\title{
Flow mechanism of polymer solution in porous media and in situ test data analysis
}

\author{
LIU Yue-wu(刘曰武) \\ (Division of Engineering Science, Institute of Mechanics, Chinese Academy of Sciences, Beijing 100080, China)
}

\begin{abstract}
A mathematical model of the polymer solution flow in porous media is established with the different concentration distribution. The polymer solution is treated as the power law non-Newtonian fluid with its power law index depended on the concentration of the solution. The finite element method is used to solve the problem by considering the effects of the concentration distributions and the different boundary conditions on the process of pressure conduction. The wellbore pressure and pressure distribution have been determined. For the different case, the type curve of pressure and its derivate have been analyzed. Some in situ test data are analyzed to verify the new model by using the type curve matching method. The results show that the concentration distribution of the polymer solution in the porous media is clearly reflected by the power law indexes changed along the distance. The characteristic parameters of the porous media and the polymer solution distribution can be determined by analyzing the in situ test data. The new model has been extended to more complicated boundary cases.
\end{abstract}

Key words: polymer solution; numerical simulation; transient flow; test data; power law index

\section{Introduction}

The tertiary recovery technology is widely applied to enhance recovery efficiency. The major tertiary recovery operations include surfactant flooding, surfactant-polymer flooding, polymer flooding, alkaline flooding and alkaline-surfactant-polymer flooding etc. Among them, only the polymer injection technology is widely used in China. There is more than $10 \times 10^{6} \mathrm{t}$ of oil produced annually by using this kind of technology.

In recent year, many scientists have focused on the issue of non-Newtonian flow in porous media ${ }^{[1-3]}$. WHITE $^{[4]}$ examined the conditions for expecting a similarity solution on the lines of Darcy's Law for non-Newtonian flow in porous media. A modified Darcy Law can be expected for a power law fluid. IKOKU ${ }^{[5]}$ gave the wellbore pressure type curve for single phase power law fluid. PASCAL ${ }^{[6]}$ presented a similarity solution for the power law fluid. $\mathrm{LIU}^{[7]}$ gave a numerical solution for polymer transient flow in the composite fluid reservoir with circle closed boundary.

In this paper, a mathematical model of the polymer solution flow in porous media is established with the different concentration distribution of the solution. The polymer solution is treated as the power law non-Newtonian fluid with its power law index depended on the concentration of the solution. The finite element method is used to solve the problem by considering the effects of the concentration distributions and the different boundary conditions on the process of pressure conduction. The wellbore pressure and pressure distribution have been determined. For the different case, the type curve of pressure and its derivate have been analyzed. Some in situ test data are analyzed to verify the new model by using the type curve matching method. Rheological parameter power law index are determined by the in situ test data.

\section{Physical model}

It supposes that the oil reservoir is homogeneous, isotropy, infinite horizontal slab formation. The polymer solution is treated as the power law non-Newtonian fluid just like that in Ref.[5]. According to the property of polymer solution and the injection process in the injection well, the concentrations of polymer solution fall into three types, i.e. the continuous distribution caused by the continuous injection, the step discontinuous distribution caused by the interim injection of polymer solution and the nonlinear distribution caused by the shear effect. The concentration distribution of polymer solution is shown in Fig.1, where $C_{0}$ and $C_{\text {end }}$ indicate the concentration of polymer solution near and far from the wellbore, respectively. The continuous distribution is the special case of the step discontinuous distribution, which have been solved in Ref.[7]. Here the last two cases will 
be discussed in this work.

For the second distribution, the power law index of the polymer solution is supposed having a step change along the distance from the polymer injection well (Fig.2(a)). The third distribution is supposed having a nonlinear change along the distance from the polymer injection well (Fig.2(b)). In Fig.2, $n_{0}$ indicates power law index of polymer solution near the wellbore.
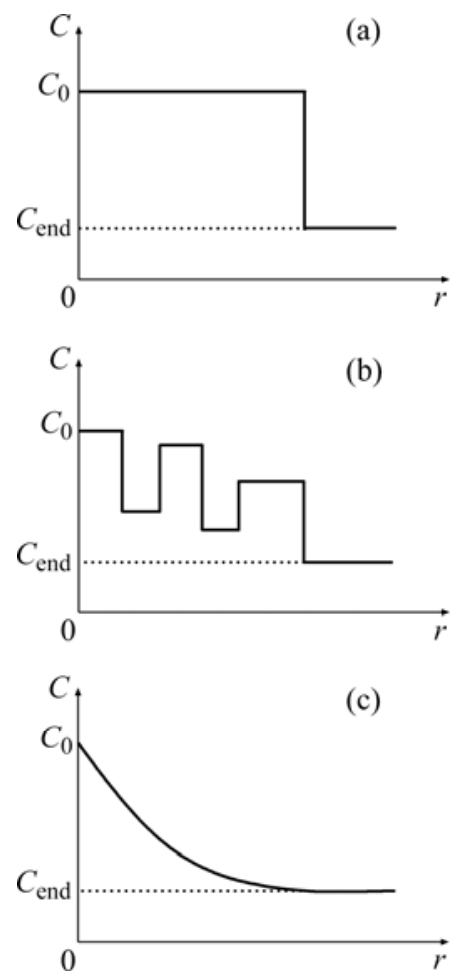

Fig.1 Concentration distribution of polymer solution along distance from injection well

(a) Continuous distribution; (b) Step distribution;

(c) Nonlinear distribution
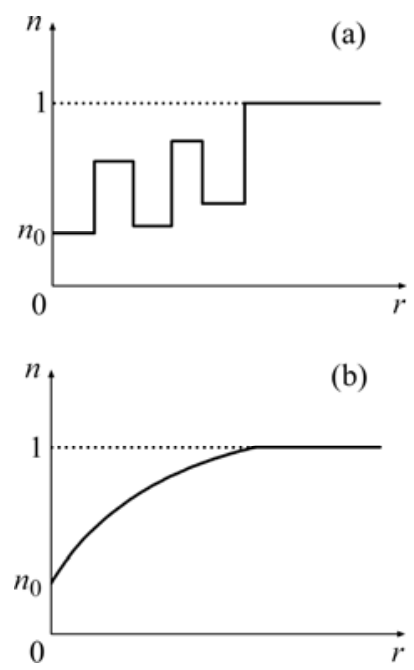

Fig.2 Change of power law index along distance from polymer injection well (a) Step change of power law index;

(b) Nonlinear change of power law index

\section{Mathematical model}

Dilute polymer solutions, however, do not possess a constant viscosity and exhibit a non-Newtonian rheological behavior. Most of them closely approximate the Ostwald de Waele power law fluid ${ }^{[3-5]}$.

Therefore, for the second polymer solution distribution, the governing equations for the polymer transient flow in the circular or infinite reservoir are as follows:

$$
\begin{gathered}
\frac{\partial^{2} p_{i \mathrm{D}}}{\partial R_{\mathrm{D}}^{2}}+\frac{n_{i}}{R_{\mathrm{D}}} \frac{\partial p_{i}}{\partial R_{\mathrm{D}}}=\frac{R_{\mathrm{D}}^{1-n_{i}}}{C_{\mathrm{D}} \mathrm{e}^{2 S}} \frac{\partial p_{i \mathrm{D}}}{\partial T_{\mathrm{D}}} \\
R_{(i-1) \mathrm{D}}<R_{\mathrm{D}}<R_{i \mathrm{D},}, \quad(i=1,2,3, \cdots, N)
\end{gathered}
$$

where $s$ is skin factor; $R_{0 \mathrm{D}}=1, R_{N \mathrm{D}}=R_{\mathrm{eD}} ; C_{D}$ is the dimensionless wellbore storage coefficient, $C_{\mathrm{D}}=1.592 \mathrm{C} /$ $\left(\phi h C_{t} r_{\mathrm{w}}\right), C$ is the wellbore storage factor; $\phi$ is the porosity of the reservoir; $h$ is the net pay of the test formation; $C_{t}$ is the total compress coefficient for the test formation; $r_{\mathrm{w}}$ is the wellbore radius; $p_{\mathrm{D}}$ is the dimensionless pressure, $p_{\mathrm{D}}=k h\left(p_{i}-p\right) /\left(1.1842 \times 10^{-3}\right.$ $q \mu B) ; k$ is the permeability of the reservoir; $p_{i}$ is the initial formation pressure; $p$ is the pressure in the formation; $q$ is the production rate; $\mu$ is the pseudo-viscosity of the fluid, $B$ is the volume factor; $R_{\mathrm{D}}$ is the dimensionless distance, $R_{\mathrm{D}}=r / r_{\mathrm{we}}, r_{\mathrm{we}}$ is the effective wellbore radius, $r_{\mathrm{we}}=r_{\mathrm{w}} \cdot \mathrm{e}^{-S} ; R_{\mathrm{eD}}$ is the dimensionless distance of the outer boundary, $R_{\mathrm{eD}}=R_{\mathrm{e}} / r_{\mathrm{we}}$, $R_{\mathrm{e}}$ is the distance of the outer boundary; $t_{\mathrm{D}}$ is the dimensionless time, $t_{\mathrm{D}}=3.6 \mathrm{kt} /\left(\phi \mu C_{t} r_{\mathrm{w}}^{2}\right) ; T_{\mathrm{D}}=t_{\mathrm{D}} / C_{\mathrm{D}} ; n_{i}$ is the power law index in region $i ; \lambda_{i}$ is the mobility ratio in region $i$.

For the third polymer solution distribution, the governing equations for the polymer transient flow in the circular or infinite reservoir are as follows:

$$
\frac{\partial^{2} p_{\mathrm{D}}}{\partial R_{\mathrm{D}}^{2}}+\frac{n_{f}}{R_{\mathrm{D}}} \frac{\partial p_{\mathrm{D}}}{\partial R_{\mathrm{D}}}=\frac{R_{\mathrm{D}}^{1-n_{f}}}{C_{\mathrm{D}} \mathrm{e}^{2 S}} \frac{\partial p_{\mathrm{D}}}{\partial T_{\mathrm{D}}}, 1<R_{\mathrm{D}}<R_{\mathrm{eD}}
$$

where $n_{f}=n_{0} \mathrm{e}^{-\beta R_{\mathrm{D}}}$.

The initial condition:

$$
p_{i \mathrm{D}}\left(R_{\mathrm{D}}, 0\right)=0, i=1,2,3, \cdots, N
$$

The inner boundary condition:

$$
\frac{\partial p_{\mathrm{wD}}}{\partial T_{\mathrm{D}}}-\left.\frac{\partial p_{1 \mathrm{D}}}{\partial R_{\mathrm{D}}}\right|_{R_{\mathrm{D}}=1}=1
$$

The closed outer boundary:

$$
\left.\frac{\partial p_{N \mathrm{D}}}{\partial R_{\mathrm{D}}}\right|_{R_{\mathrm{D}}=R_{\mathrm{eD}}}=0
$$


The constant pressure boundary:

$$
\left.p_{N \mathrm{D}}\right|_{R_{N \mathrm{D}}=R_{\mathrm{eD}}}=0
$$

The infinite boundary:

$$
\left.p_{N \mathrm{D}}\right|_{R_{N \mathrm{D} \rightarrow \infty}}=0
$$

Interface pressure boundary conditions:

$$
p_{(i-1) \mathrm{D}}\left(R_{i \mathrm{D}}, T_{\mathrm{D}}\right)=p_{i \mathrm{D}}\left(R_{i \mathrm{D}}, T_{\mathrm{D}}\right), i=2,3, \cdots, N-1
$$

Flow rate continue interface condition:

$$
\begin{gathered}
R_{\mathrm{D}}^{1-n_{i-1}} \frac{\partial p_{(i-1) \mathrm{D}}\left(R_{i \mathrm{D}}, T_{\mathrm{D}}\right)}{\partial R_{\mathrm{D}}}=\lambda_{i} R_{\mathrm{D}}^{1-n_{i}} \frac{\partial p_{i \mathrm{D}}, T_{\mathrm{D}}}{\partial R_{\mathrm{D}}} \\
i=2,3, \cdots, N-1
\end{gathered}
$$

\section{Numerical simulation}

It's difficult to get analytical solution from Eqns.(1) and (2), so the FEM is selected to solve the polymer transient flow problem. In order to use the FEM, it needed to divide the research region into a large number of elements. According to the mesh automatic generation method given in the Refs.[8-9], the meshes of the reservoir showed in Fig.1 are generated in Fig.2. Then, the finite element equations for every unit in the calculated region are shown as follows:

$$
\begin{gathered}
\iint_{A} \varphi_{j n}^{\mathrm{e}}\left(\frac{\partial^{2} p_{i \mathrm{D}}^{\mathrm{e}}}{\partial R_{\mathrm{D}}^{2}}+\frac{n_{i}}{R_{\mathrm{D}}} \frac{\partial p_{i \mathrm{D}}^{\mathrm{e}}}{\partial R_{\mathrm{D}}}-\frac{R_{\mathrm{D}}^{1-n_{i}}}{R_{\mathrm{D}}} \frac{\partial p_{i \mathrm{D}}^{\mathrm{e}}}{\partial T_{\mathrm{D}}}\right) \mathrm{d} A=0 \\
R_{(j-i) \mathrm{D}}<R_{\mathrm{D}}<R_{j \mathrm{D}}, i=1,2, \cdots, N, j=1,2,3 \\
\iint_{A} \varphi_{j n}^{\mathrm{e}}\left(\frac{\partial^{2} p_{\mathrm{D}}^{\mathrm{e}}}{\partial R_{\mathrm{D}}^{2}}+\frac{n_{f}}{R_{\mathrm{D}}} \frac{\partial p_{\mathrm{D}}^{\mathrm{e}}}{\partial R_{\mathrm{D}}}-\frac{R_{\mathrm{D}}^{1-n_{f}}}{R_{\mathrm{D}}} \frac{\partial p_{\mathrm{D}}^{\mathrm{e}}}{\partial T_{\mathrm{D}}}\right) \mathrm{d} A=0 \\
1<R_{\mathrm{D}}<R_{\mathrm{eD}}, j=1,2,3
\end{gathered}
$$

where $\varphi_{i}^{\mathrm{e}}$ is the interpolating function; $p_{\mathrm{D}}^{\mathrm{e}}$ is the pressure at every node of the calculating element.

The transient flow problem for the low permeability reservoir with the composite start-up pressure gradients and the mobility radios is solved. The finite element equations of the calculating elements for the inner and outer region are derived:

$$
\begin{gathered}
A\left(b_{i}^{2}+c_{i}^{2}+\frac{C S T_{\mathrm{D}}}{6}\right) p_{i}^{\mathrm{e}, n+1}+ \\
A\left(b_{i} b_{j}+c_{i} c_{j}+\frac{C S T_{\mathrm{D}}}{12}\right) p_{j}^{\mathrm{e}, n+1}+ \\
A\left(b_{i} b_{k}+c_{i} c_{k}+\frac{C S T_{\mathrm{D}}}{12}\right) p_{k}^{\mathrm{e}, n+1}= \\
\operatorname{CST}_{\mathrm{D}} \cdot \frac{A}{12}\left(2 p_{i}^{\mathrm{e}, n}+p_{j}^{\mathrm{e}, n}+p_{k}^{\mathrm{e}, n}\right)-\frac{L}{2}
\end{gathered}
$$

where $\quad C S=1 /\left(C_{D} \mathrm{e}^{2 S}\right), A=\frac{1}{2}\left|\begin{array}{ccc}1 & 1 & 1 \\ x_{i} & x_{j} & x_{k} \\ y_{i} & \mathrm{y}_{\mathrm{j}} & y_{k}\end{array}\right|$,

$$
\begin{aligned}
& a_{i}=\frac{1}{2}\left(x_{j} y_{k}-x_{k} y_{j}\right), b_{i}=\frac{1}{2 A}\left(y_{j}-y_{k}\right), \\
& c_{i}=\frac{1}{2 A}\left(x_{k}-x_{j}\right)
\end{aligned}
$$

By using the FEM, the pressure distribution can be determined in the formation near the polymer injection well. Fig.3(a) shows the pressure profile near the wellbore. Fig.3(b) shows the pressure distribution in the formation near the polymer injection well.
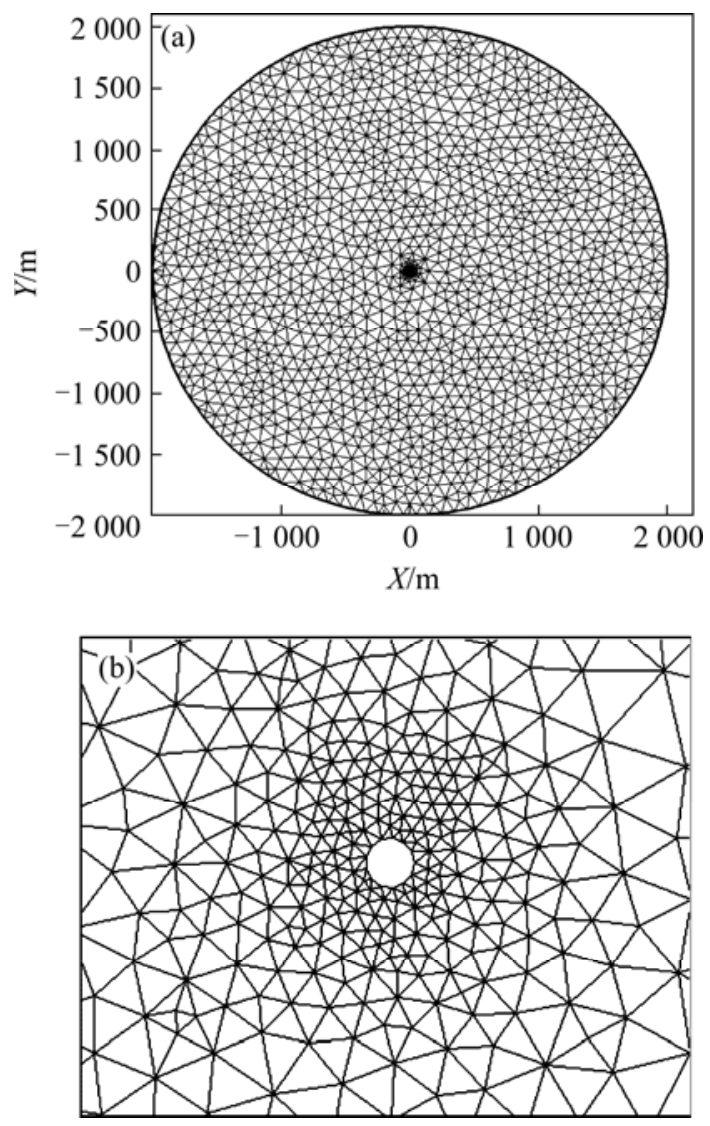

Fig.3 Mesh of research region

(a) Mesh of whole region; (b) Mesh near wellbore

The wellbore transient pressure typical curves are got by FEM in the same process. The effects of non-Newtonian power law index and the boundary distance have been considered. The results of pressure distribution in research area is shown in Fig.4. The type curve is shown in Fig.5, which is the case for the reservoir formation with circle closed boundary.

From Fig.5, it shows that for the different stage the pressure and pressure curve have different shape features. At the early stage, both the pressure and its derivative 

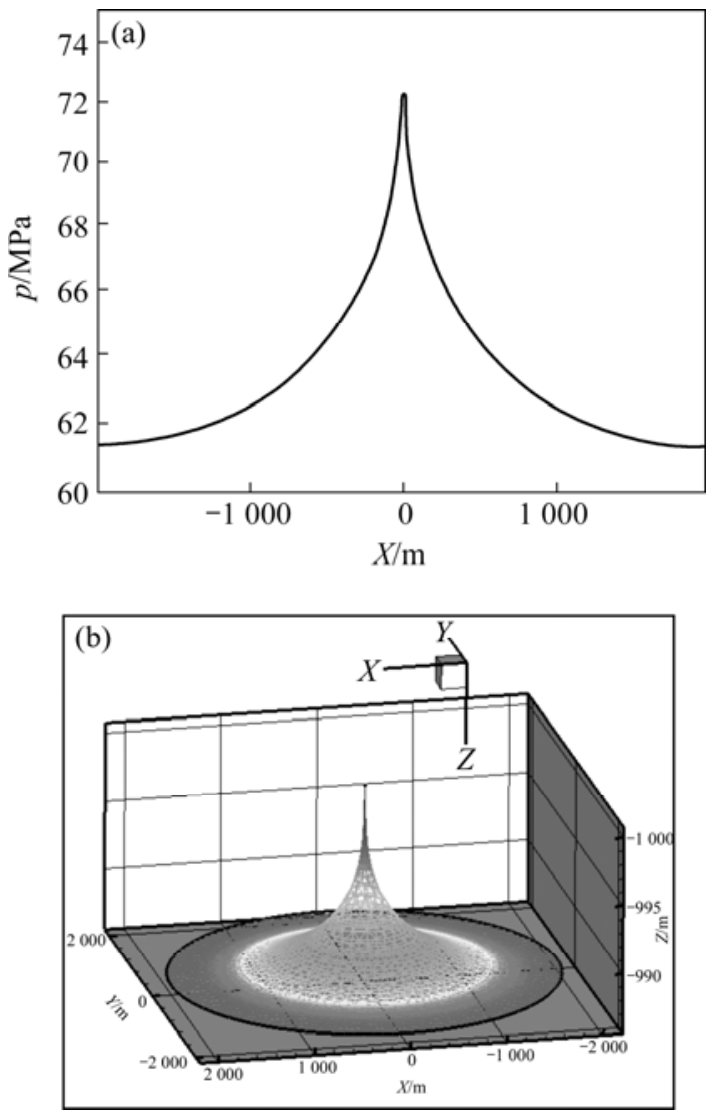

Fig.4 Pressure distribution in research area

(a) Pressure profile near wellbore;

(b) Pressure distribution in formation

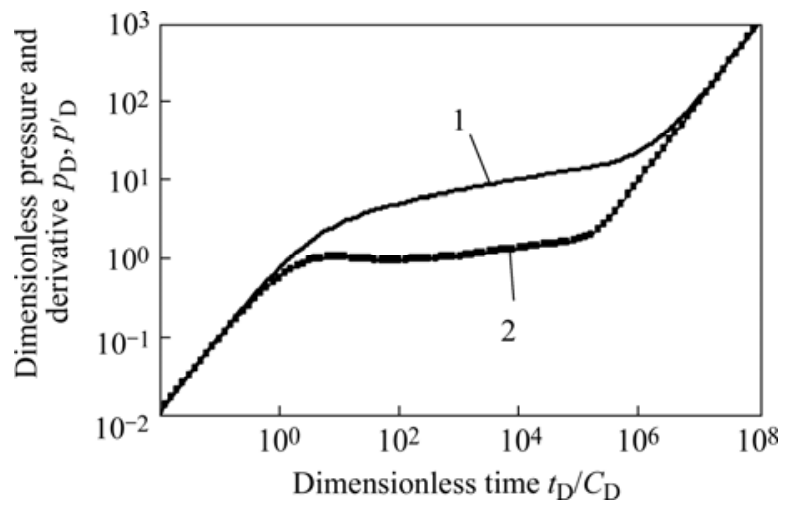

Fig.5 Wellbore pressure type curves

1-Pressure curve; 2 - Pressure derivate curve

curve are straight line with slope equal to 1 , it indicates that the fluid flow in the reservoir formation is controlled by the wellbore storage. In the next stage, there is a swell in the pressure derivative curve, that means there must exist some formation damage near the wellbore. After then the pressure derivative curve holds up, the affect of non-Newtonian fluid character becomes distinctly. If the power law index is a constant, the pressure curve may parallel to its derivative curve. Otherwise the pressure derivative will change in a nonlinear form and the two curves are not paralleled. In the last stage, both the pressure and its derivative curve are straight line with slope equal to 1 , which reflects the effect of the circle closed boundary.

\section{Comparison}

The new model can be simplified to solve the transient flow problem in a single connected region. If the outer boundary is large enough, the transient wellbore pressure typical curve in $\log -\log$ form can be got just like the analytical solution of $\mathrm{IKOKU}^{[5]}$. In that case, the non-Newtonian power law index $n \neq 1$, the theoretical pressure and pressure derivative curves are shown in Fig.5.

If the value of the power law index is equal to 1 , the value of the pressure derivate curve is equal to 0.5 , namely the Newtonian transient flow case. The comparison of the special numerical solution with the analytical solution for the circular closed boundary is just shown in Fig.6. The new numerical model can be expended to the other boundary conditions such as infinite boundary and circular constant pressure boundary.

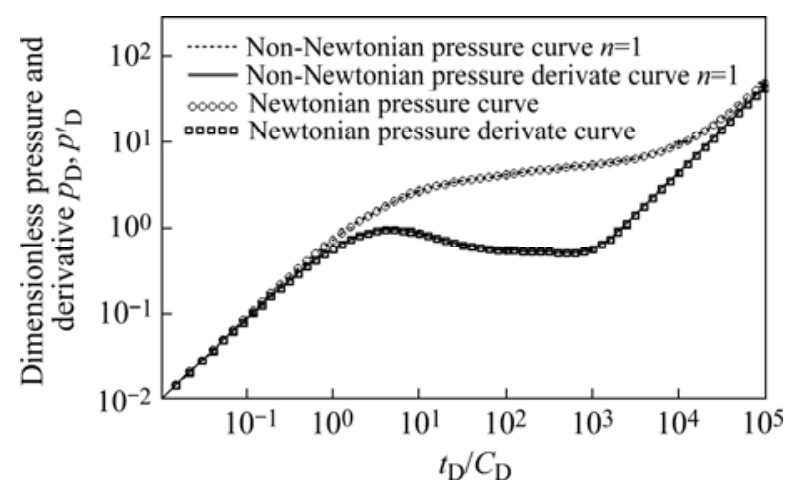

Fig.6 Results compared with those of the Newtonian fluid

\section{Test data analysis}

Since the power law index of the polymer solution in the formation is different from that in the surface, the test must execute in the formation condition. In situ test performed by using a pressure gauge at the depth of the injection formation in the wellbore during the polymer solution injecting process. Some field in situ tests have been analyzed by using type curve matching method based on the model developed in this paper. One of them is shown in Fig.7. The change of the power law index of this example is $0.95 \exp \left(-0.3 R_{D}\right)$, which is shown in Fig.8. 


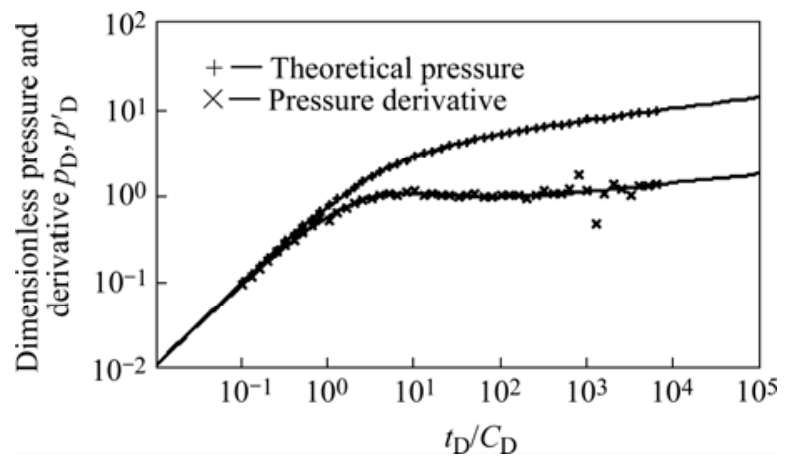

Fig.7 Field test data matching curves

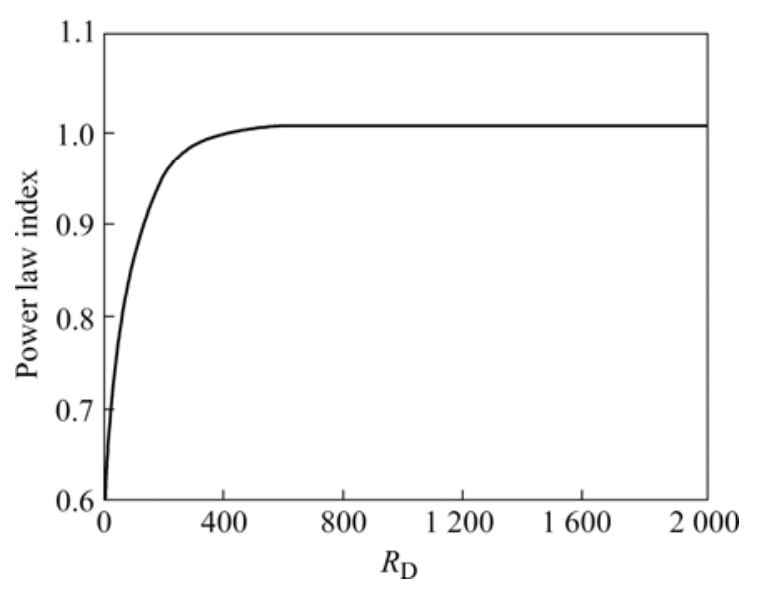

Fig.8 Chang of power law index along distance

\section{Summary}

A mathematical model of the polymer solution flow in porous media is established with the different concentration distribution of the solution. The polymer solution is treated as the power law non-Newtonian fluid with its power law index depended on the concentration of the solution. The finite element method is used to solve the problem by considering the effects of the concentration distributions and the different boundary conditions on the process of pressure conduction. Some in situ test data are analyzed to verify the new model by using the type curve matching method. The results show that the concentration distribution of the polymer solution in the porous media is clearly reflected by the power law indexes changed along the distance. The characteristic parameters of the porous media and the polymer solution distribution can be determined by analyzing the in situ test data.

\section{References}

[1] XAVIER L, VALVATNE P H, MARTIN J B. Predictive network modeling of single-phase non-Newtonian flow in porous media[J]. Journal of Colloid and Interface Science, 2003, 264(1): 256-265.

[2] PEARSON J R A, TARDY P M J. Models for flow of non-Newtonian and complex fluids through porous media[J]. Journal of Non-Newtonian Fluid Mechanics, 2002, 102(2): 447-473.

[3] SANTOYO E, SANTOYO-GUTIÉRREZ S, GARCíA A, et al. Rheological property measurement of drilling fluids used in geothermal wells[J]. Applied Thermal Engineering, 2001, 21(3): 283-302.

[4] WHITE D A. Non-newtonian flow in porous media[J]. Chemical Engineering Science, 1967, 22(4): 669-672.

[5] IKOKU C U, RAMEY H J. Transient flow of non-Newtonian power law fluids in porous media[J]. J Soc Pet Eng, 1979, 44(3): 164-174.

[6] PASCAL H. Similarity solutions to some unsteady flows of non-Newtonian fluids of power law behavior[J]. International Journal of Non-Linear Mechanics, 1992, 27(5): 759-771.

[7] LIU Yue-wu, CHEN Hui-xin, GONG Xin, et al. Numerical solution for polymer transient flows in a circle bounded composite formation $[\mathrm{C}] / /$ Proceedings of the Fourth International Conference on Fluid Mechanics. Beijing: Tsinghua University Press, 2004; 145-148.

[8] WILSON E L. Automation of the finite element method-A personal historical view [J]. Finite Elements in Analysis and Design, 1993,13(2): 91-104.

[9] ZHU J Z. A new approach to the development of automatic quadrilateral mesh generation $[\mathrm{J}]$. International Journal for Numerical Methods in Engineering, 1991, 32(8): 849-866.

(Edited by ZHAO Jun) 